\title{
Las aprehensiones en el registro gráfico para el estudio de la derivada parcial
}

\author{
KATIA VIGO INGAR* \\ Pontificia Universidad Católica del Perú - Perú \\ MARIA JOSÉ FERREIRA DA SILVA* \\ Pontificia Universidad Católica de São Paulo - Brasil \\ Recibido el 06-03-17; primera evaluación el 25-07-18; \\ segunda evaluación el 06-03-19; tercera evaluación el \\ 22-03-19; aceptado el 27-03-19
}

\section{RESUMEN}

El objetivo de este artículo es presentar una extensión del estudio de Duval en relación con las aprehensiones en el registro gráfico de una función de dos variables, analizar una situación didáctica e investigar las articulaciones de las aprehensiones en el estudio de la derivada parcial. Su pertinencia en la enseńanzaaprendizaje del Cálculo Diferencial de dos variables es amplia, puesto que la información que el gráfico proporciona es importante para la construcción de conocimientos de funciones de dos variables. Nuestra investigación es cualitativa, concretamente, aspectos de la Ingeniería Didáctica de Michèle Artigue. Se constató que la articulación de las aprehensiones en el registro gráfico, mediado por el software Mathematica, permitió a los alumnos conjeturar propiedades, aplicarlas en problemas de optimización y adaptarse a resolver geométricamente dichos problemas.

Palabras clave: aprehensiones en el registro gráfico; visualización; ingeniería didáctica; situación didáctica; derivada parcial.

\footnotetext{
* Licenciada en Matemática por la Universidad Nacional de Trujillo, Perú. Magíster en Docencia Universitaria por la Universidad Nacional del Callao, Perú. Magíster en Matemática por la Pontificia Universidad Católica del Perú. Doctora en Educación Matemática por la Pontificia Universidad Católica de São Paulo, Brasil. Línea de Investigación: Tecnologías y Visualización en Educación Matemática. Correo electrónico: kvigo@pucp.pe

** Licenciada en Matemáticas. Magíster en Enseñanza de las Matemáticas. Doctora en Educación Matemática por la Pontificia Universidad Católica de São Paulo, Brasil. Profesora en el Departamento de Matemáticas y en el Programa de Posgrado en Educación Matemática. Participa en el grupo de investigación PEA-MAT (Procesos de enseñanza y aprendizaje de las matemáticas). Ganadora de la Cátedra Profesor Visitante José Tola Pasquel 2016. Correo electrónico: zeze@pucsp.br
} 


\section{The apprehensions in the graphic registry for the study of the partial derivative}

\section{Abstract}

The objective of this article is to present an extension of the Duval study in relation to the apprehensions in the graphic register of a function of two variables, to analyze a didactic situation and to investigate the articulations of the apprehensions in the study of the partial derivative. Its relevance in the teaching-learning of the Differential Calculus of two variables is wide, since the information that the graph provides is important for the construction of knowledge of functions of two variables. Our research is qualitative, specifically, aspects of the didactic engineering of Michèle Artigue. It was found that the articulation of the apprehensions in the graphic registry, mediated by the Mathematica software, allowed the students to conjecture properties, apply them in optimization problems and adapt to solve these problems geometrically.

Keywords: apprehensions in the graphic register; display; didactic engineering; didactic situation; partial derivative.

\section{Apreensóes no registro gráfico para o estudo da derivada parcial}

\section{Resumo}

O objetivo deste artigo é apresentar uma extensão do estudo de Duval em relação às apreensôes no registro gráfico de uma função de duas variáveis, analisar uma situaçáo didática e investigar as articulaçóes das apreensóes no estudo da derivada parcial. Sua relevância no ensino-aprendizagem do Cálculo Diferencial de duas variáveis é ampla, uma vez que as informaçóes que o gráfico fornece é importante para a construção do conhecimento de funções de duas variáveis. Nossa pesquisa é qualitativa, especificamente, aspectos da engenharia didática de Michèle Artigue. Constatou-se que a articulaçáo das apreensóes no registro gráfico, mediada pelo software Mathematica, permitiu que os alunos conjecturassem propriedades, aplicassem-nas em problemas de otimização e se adaptassem para solucionar esses problemas geometricamente.

Palavras-chave: apreensōes no registro gráfico; visualização; engenharia didática; situação didática; situação didática; derivada parcial. 


\section{INTRODUCCIÓN}

En las dos últimas décadas, el estudio de las funciones de dos variables está teniendo un progresivo desarrollo, como muestran los trabajos de Yerushalmy (1997), Kabael (2009), Montiel, Wilhelmi, Vidakovic y Elstak (2009), y Trigueros y Martínez-Plannell (2010, 2011), desde los marcos teóricos de la teoría APOS (Acción, Proceso, Objeto, Esquema), Registros de Representación Semiótica (RRS), el enfoque Ontosemiótico y la Teoría Antropológica de lo Didáctico (TAD). Respecto al proceso de visualización, tenemos a Zimmermann y Cunningham (1991) en el área del cálculo en dos variables, a Guzmán (1996) en el área del Análisis y a Duval (1999) en la Geometría.

Según estos investigadores y basados en su práctica docente, se afirma que, en el curso de cálculo de varias variables, resolver un problema de optimización significa aplicar dos teoremas de manera mecánica, algebraica y solo teniendo en cuenta funciones polinomiales diferenciables. Asimismo, se pone muy poco énfasis en el uso de la representación gráfica para resolver un problema, o en un análisis de los resultados, es decir, no se permite que el estudiante desarrolle su proceso de visualización.

Para Zimmermann y Cunningham (1991), el término visualización describe el proceso de producción o el uso de representaciones geométricas o gráficas de conceptos matemáticos, principios o problemas, por medio de un diseńo a mano o generado por computador. Según los autores, las ciencias, la ingeniería y las matemáticas están disfrutando un renacimiento del interés en la visualización. Es más, afirman lo siguiente:

En la visualización, lo que interesa es precisamente la capacidad del estudiante para diseñar un diagrama apropiado (con lápiz y papel o, en algunos casos, con un computador) para representar un concepto matemático o problema y utilizar un diagrama para la obtención de la comprensión, y como una ayuda en la resolución de problemas (p. 3).

En la misma línea, y haciendo énfasis en el modo de producción de esas representaciones, Guzmán (1996) afirma lo siguiente:

Las ideas, conceptos y métodos de la matemática presentan una gran riqueza de contenidos visuales, representables intuitivamente, geométricamente, cuya utilización resulta muy provechosa, tanto en las tareas de presentación y manejo de tales conceptos, y métodos como en la manipulación con ellos para la resolución de problemas del campo (p. 1). 
Duval $(1994,1995)$ trata el aprendizaje de la geometría. Para comprenderla, desarrolló la idea de aprehensiones y del desarrollo de maneras de ver. La teoría del aprendizaje de la matemática en Duval (1995, 1996, 1999, 2003 , 2004) tiene como base los registros de representación semiótica. Para el caso de la geometría, el autor sugiere tipos de aprehensión en la resolución de problemas. Ingar (2014) hace una extensión del estudio de Duval (1999) en relación con las aprehensiones en el registro gráfico de una función de dos variables.

En este trabajo, adoptaremos la visualización como una noción fundamental para la comprensión de la matemática, en vista de que Duval (1999), único estudio, afirma que no hay comprensión sin visualización, puesto que ella permite una aprehensión completa de cualquier organización de relaciones entre unidades de representación. En el caso de la funciones de dos variables, cuando tratamos de comprender su representación en el registro gráfico, esas unidades llamadas "variables visuales» serían el eje de la superficie y los cortes en el plano $x y$, la intersección con el origen de coordenadas y los cortes con los planos coordenados (Ingar y Silva, 2015). Por ello, nos propusimos estudiar la visualización del registro gráfico de ese tipo de funciones, pues, de acuerdo con Kashefi, Zaleha y Mohammad (2010), la falta de su comprensión causará obstáculos en el aprendizaje de otros conceptos que involucren este tipo de función. Además, en la mayoría de los libros de texto utilizados por los estudiantes, la representación de funciones de dos variables en el registro gráfico tiene como propósito solo ilustrar el gráfico de la función, pero no son explicados los procedimientos que permiten la visualización de dicha función.

Es así que, el propósito del artículo es de presentar una extensión del estudio de Duval en relación con las aprehensiones en el registro gráfico de una función de dos variables, analizar una situación didáctica e investigar las articulaciones de las aprehensiones en el registro gráfico en el Sistema Algebraico Computacional $(C A S)$ que realizaron los estudiantes de ingeniería del segundo año de estudios en el aprendizaje de la derivada parcial y su uso, para encontrar el valor máximo local de una función de dos variables.

Con respecto al software, el CAS Mathematica, los estudiantes utilizaron la versión 8 , dado que estaba instalado en el laboratorio de cómputo, escenario donde se realizó la experimentación. Más aún, este $C A S$ permite la manipulación en el registro gráfico en tres dimensiones y facilita el proceso que lleva a la visualización de un representante de la función de dos variables en el registro gráfico.

El uso del Mathematica provocó cambio de hábitos del estudiante respecto al trabajo y al aprendizaje de la matemática, pues el objeto matemático vive en el ambiente tecnológico de manera diferente que en el ambiente lápiz y papel. 


\section{MarCo teórico}

Según Duval (2004), las representaciones gráficas cartesianas son muy utilizadas, debido a que no solamente son encontradas en los manuales o artículos, sino también en las revistas. Esos gráficos pueden ser vistos de dos maneras: una puntual, el cual indica un valor en un momento dado; y otra icónica, que evoca lo alto y lo bajo, las subidas suaves o abruptas a partir de un nivel de base. Sin embargo, para el autor, ninguna de esas corresponde a la manera útil de ver desde el punto de vista matemático, es decir, existe una tercera forma que permite visualizar una relación entre las unidades significantes del registro algebraico y las unidades visuales del registro gráfico.

El autor afirma que distinguimos lo que se observa en el gráfico cartesiano y lo que los aspectos observados permiten identificar. Uno de los problemas específicos del aprendizaje es ¿cómo hacer pasar a los estudiantes de una aprehensión local e icónica a una aprehensión global cualitativa?, pues solamente con este último tipo de aprehensión es que podemos hacer la coordinación con el registro algebraico y sus relaciones con el registro gráfico, pudiendo así los gráficos cartesianos funcionar como herramientas en el proceso de visualización.

Visualizar un gráfico requiere una interpretación de una representación gráfica. Según el autor, para esa interpretación, es importante la discriminación de las variables visuales, exponiendo esa representación a todas las variaciones posibles, con la condición de que las formadas de ese modo continúen teniendo sentido. Así, buscamos en Duval (1994), la noción de aprehensión de una figura geométrica para estudiar y comprender esas posibles variaciones en el registro gráfico, particularmente en el registro gráfico de funciones de dos variables reales, cuando construimos el concepto y propiedades de los valores extremos locales.

\subsection{Aprehensión en el registro gráfico de funciones de dos variables}

Se usa el término «aprehender» en el sentido de comprender a la perfección la información, asimilándola y pudiendo reutilizarla a partir de la interpretación propia.

Existen cuatro formas de aprehender una figura: la perceptiva, la discursiva, la secuencial y la operatoria. La aprehensión perceptiva es aquella que permite identificar o reconocer una forma o un objeto matemático. Para el investigador Duval, «la aprehensión perceptiva tiene la función epistemológica de identificación de los objetos en dos o tres dimensiones. Esto es hecho por procesos cognitivos efectuados automáticamente, y así, de forma inconsciente» (Duval, 1994, p. 124). 
Respecto a la representación de una función de dos variables en el registro gráfico, Ingar (2014) establece que la aprehensión perceptiva del gráfico $C A S$ (representación de una función de dos variables en el registro gráfico utilizando el Mathematica), por ejemplo, mostrado en la figura 1, permite identificar un paraboloide cumpliendo la función epistemológica de identificación de objetos.

Figura 1. Aprehensión perceptiva de un registro gráfico CAS

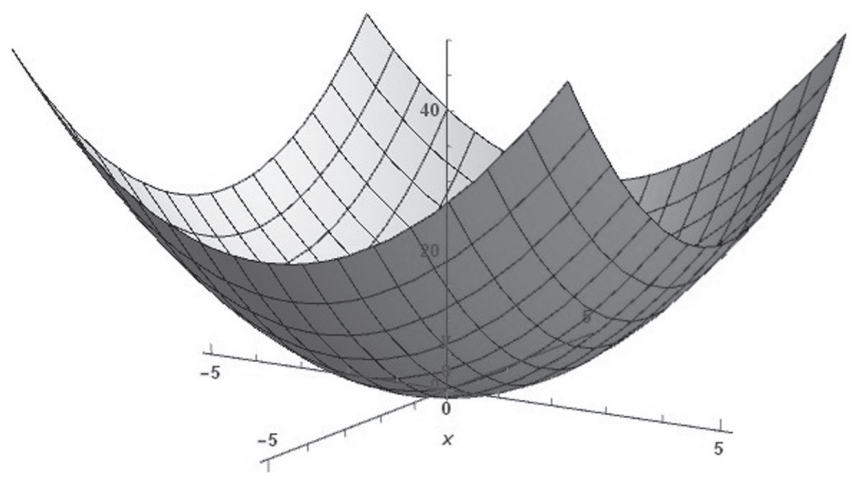

Desde el punto de vista cognitivo, la autora afirma que esa aprehensión requiere más del estudiante que lo que requiere la aprehensión perceptiva del cuadrado. Por ejemplo, debido a que en este registro gráfico se observa, además de los ejes coordenados, el tipo de variable (dos independientes y una dependiente), los valores de las variables $x, y$, $z$, la lectura de los ejes coordenados y las puntas, lo que nos permite aseverar que la aprehensión perceptiva del registro gráfico $C A S$ es más compleja que la aprehensión perceptiva de una figura en la geometría. Esta afirmación es más evidente, según la autora, cuando la aprehensión perceptiva del gráfico $C A S \_M A T H$-mostrado en la figura 2- permite identificar las mismas características anteriormente mencionadas, pero requiere más recursos cognitivos por parte del estudiante, como, por ejemplo, la construcción del sistema cartesiano $\mathrm{R}^{3}$, propio del software Mathematica, cuya representación no es la canónica.

Con respecto a que los gráficos muestran "puntas», los estudiantes no perciben que estos (gráficos) sean truncados por planos, cuyas representaciones son invisibles y determinados lateralmente por los extremos del dominio de la función representado en el Mathematica, y superiormente por el valor máximo de la función en ese dominio. De ahí que coincidimos con Trigueros y Martínez (2010) cuando afirma que la comprensión de gráficos y construcción del concepto de funciones de dos variables no son fáciles para 
los estudiantes, además de la interpretación de gráficas y la determinación de su dominio y rango.

Figura 2. Aprehensión perceptiva de un registro gráfico CAS_MATH

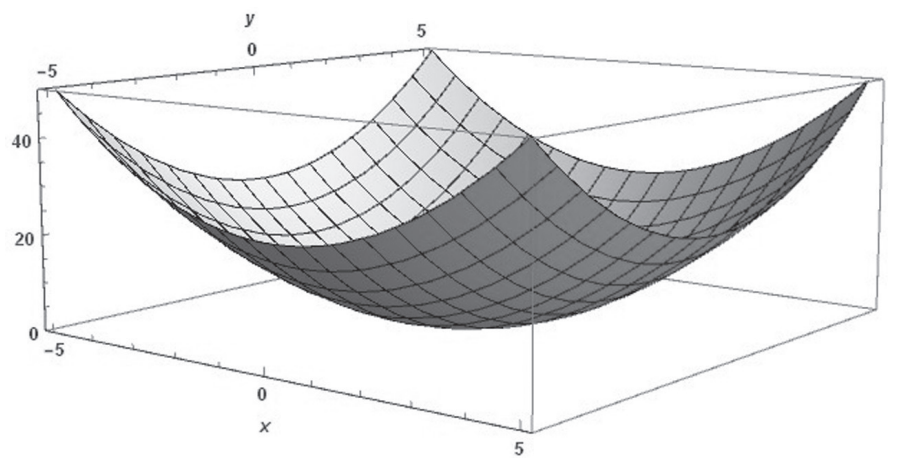

La aprehensión discursiva de una figura, según Duval (1994), explica propiedades matemáticas de la figura, como aquellas indicadas por una leyenda o por las hipótesis, que se configura como explicaciones de naturaleza deductiva con la función epistemológica de demostración. En la representación de una función de dos variables en el registro gráfico, Ingar (2014) afirma que la aprehensión discursiva describe propiedades matemáticas del registro gráfico, considerando la semántica de las propiedades del objeto con la función de formular conjeturas para la construcción del conocimiento. A modo de ejemplo, en la figura 3, basado de Thomas (2005), describimos las propiedades del valor máximo local en el punto $\left(x_{0}, y_{0}\right)$.

Figura 3. Aprehensión discursiva del registro gráfico

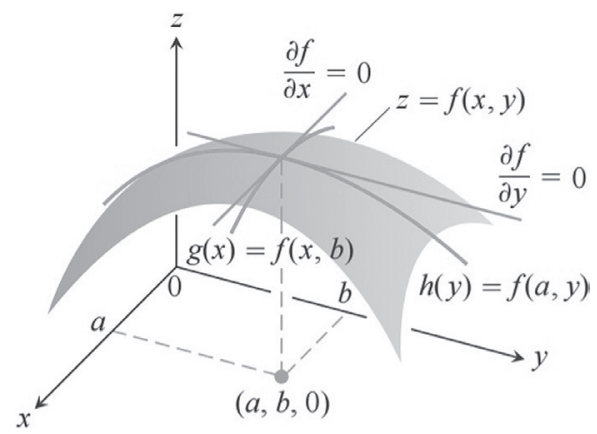

Fuente: Thomas (2005)

$f(x, y)$ tiene un máximo local, un punto interior $(a, b)$ de su dominio y las derivadas parciales de la función, representada por $z=f(x, y)$ existen en $(a, b)$, las curvas representadas por $g(x)=f(x, b)$ tiene un extremo local en $x=a$, y $h(y)=f(a$, $y$ ) tiene extremo local en $y=b$, luego $f_{x}(a, b)=0$ y $f_{v}(a, b)=0$. 
La aprehensión secuencial, según Duval (1994), es requerida siempre que se desea construir una figura o describir su construcción, y trata del orden de construcción de una figura. Ese orden no solo depende de las propiedades matemáticas de la figura, sino también de las herramientas técnicas utilizadas (la regla y el compás).

En la representación de una función de dos variables en el registro gráfico, Ingar (2014) afirma que la aprehensión secuencial es una secuencia de pasos para graficar una función y resalta que si representamos la función en el registro gráfico con apoyo de un software por el ejemplo, para construir el registro gráfico $C A S$ o el registro gráfico $C A S \_M A T H$, no solo precisamos conocer las herramientas técnicas utilizadas, es decir, el menú de comandos del software y su sintaxis, sino también requerimos los conocimientos matemáticos del usuario para estar en continua dialéctica con las representaciones semióticas propias del software. Precisamente, las instrucciones del paso 1 al paso 5 repasadas y exploradas, explícitamente, favorecerían la comprensión del concepto de función relacionado a su dominio y rango, por ejemplo.

Es decir, conforme se muestra a continuación, escribimos los comandos necesarios del software Mathematica para descubrir secuencialmente y localizar el mínimo local en el registro gráfico $C A S \_M A T H$ de una función de dos variables (figura 4).

Paso 1: Escribir el comando,

Plot $3 D[\operatorname{Sen}[x]+\operatorname{Sin}[y]+\operatorname{Sin}[x+y],\{x, 0,7\},\{y, 0,7\}$, Axeslabel $\rightarrow$

$\left\{\mathrm{X}^{\prime \prime}, \mathrm{Y}^{\prime \prime}\right\}$, LabelStyle $\rightarrow$ Directive [Blue, Bold], AxesOrigin $\rightarrow$

$\{0,0,0\}$, Boxed $\rightarrow$ False, PlotTheme $\rightarrow$ Scientific, AxesStyle $\rightarrow$

Directive $[$ Blue,12]], para graficar una función de dos variables.

Paso 2: Escribir el comando,

ContourPlot $3 D[z==-3,\{x, 2,8\},\{y, 2,8\},\{z,-6,2\}$, Axeslabel $\rightarrow$ $\left\{\mathrm{X}^{\prime \prime}, \mathrm{Y}^{\prime \prime}\right\}$, LabelStyle $\rightarrow$ Directive [Blue, Bold], AxesOrigin $\rightarrow$ $\{0,0,0\}$, Boxed $\rightarrow$ False, PlotTheme $\rightarrow$ "Classic", AxesStyle $\rightarrow$ Directive[Blue,12], Mesh $\rightarrow$ None], para graficar el plano horizontal representado por $z=-3$. 
Paso 3: Escribir el comando

ContourPlot $3 D[z==-2,7,\{x, 2,8\},\{y, 2,8\},\{z,-6,2\}$, Axeslabel $\rightarrow$ $\left\{\mathrm{X}^{\prime \prime}, \mathrm{Y}^{\prime \prime}\right\}$, LabelStyle $\rightarrow$ Directive [Blue, Bold], AxesOrigin $\rightarrow$ $\{0,0,0\}$, Boxed $\rightarrow$ False, PlotTheme $\rightarrow$ "Web", AxesStyle $\rightarrow$ Directive[Blue,12], Mesh $\rightarrow$ None], para graficar el plano horizontal representado por $z=-2,7$.

Paso 4: Escribir el comando

ContourPlot $3 D[z==-2,4,\{x, 2,8\},\{y, 2,8\},\{z,-6,2\}$, Axeslabel $\rightarrow$ $\left\{\mathrm{X}^{\prime \prime}, \mathrm{Y}^{\prime \prime}\right\}$, LabelStyle $\rightarrow$ Directive [Blue, Bold], AxesOrigin $\rightarrow$ $\{0,0,0\}$, Boxed $\rightarrow$ False, PlotTheme $\rightarrow$ "Classic", AxesStyle $\rightarrow$ Directive[Blue,12], Mesh $\rightarrow$ None], para graficar el plano horizontal representado por $z=-2,4$.

Paso 5: Escribir el comando Show $[S 1, P 1, P 2, P 3]$ para representar en un único gráfico tanto la superficie, $\mathrm{S} 1$, como el conjunto de planos, $\{\mathrm{P} 1, \mathrm{P} 2, \mathrm{P} 3\}$, hasta tener la aprehensión perceptiva del posible valor mínimo. Hecho que sucede cuando el plano horizontal es tangente a la función de dos variables.

Figura 4. Aprehensión secuencial del mínimo absoluto de una función de dos variables.

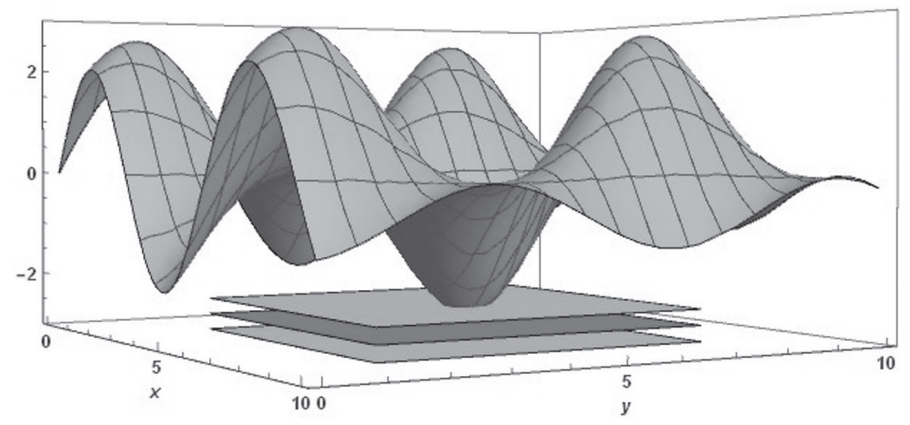

Se observa en la Figura 4 que la aprehensión secuencial de construcción de planos horizontales muestra un patrón, lo que permite conjeturar que la función de dos variables representada algebraicamente por $f(x, y)=\operatorname{sen} x+$ $\operatorname{seny}+\operatorname{sen}(x+y)$ tiene un mínimo absoluto en el punto $(0,0)$, puesto que 
por la aprehensión perceptiva, el plano representado por $z=0$ es tangente a la superficie en ese punto.

Para Duval (1994), la aprehensión operatoria corresponde a la transformación y modificación de una figura inicial en otras posibles figuras y en la organización perceptiva de esas modificaciones para mostrar la idea de una solución de una determinada situación problema. Su función es de exploración heurística, porque frecuentemente la figura geométrica es transformada en otras para mostrar una idea de la solución de un problema o una demostración. El autor distingue tres tipos de modificaciones que pueden ser de la misma forma y orientación, pero con variación de tamaño (modificaciones ópticas); del mismo tamańo y forma, pero con variación de orientación: rotación, traslación (modificaciones posicionales); y de descomposición y recomposición (modificaciones mereológicas), buscando reciprocidad entre el diseño y la representación mental.

En la representación de una función de dos variables en el registro gráfico, Ingar (2014) afirma que la aprehensión operatoria en el registro gráfico es la transformación o modificación de un gráfico inicial en otros gráficos para mostrar la idea de una solución de un problema con la misma función de exploración heurística, porque permite elaborar conjeturas para resolver el problema. La autora afirma que esta exploración heurística es posible con el apoyo del software, puesto que este permite el dinamismo del registro gráfico, lo que no es posible con el gráfico hecho con lápiz y papel, sobre todo cuando se grafica funciones de dos variables.

Ingar (2014) distingue tres modificaciones: óptica, posicional y mereológica. La modificación óptica implica variación de tamaño y constancia de forma. Ocurre cuando, presionando la tecla Ctrly clicando el botón izquierdo del mouse, movemos físicamente el mouse y se manipula el gráfico, de tal manera que se amplía o se reduce. Por ejemplo, en la figura 5, tenemos la ampliación del gráfico.

Figura 5. Modificación óptica

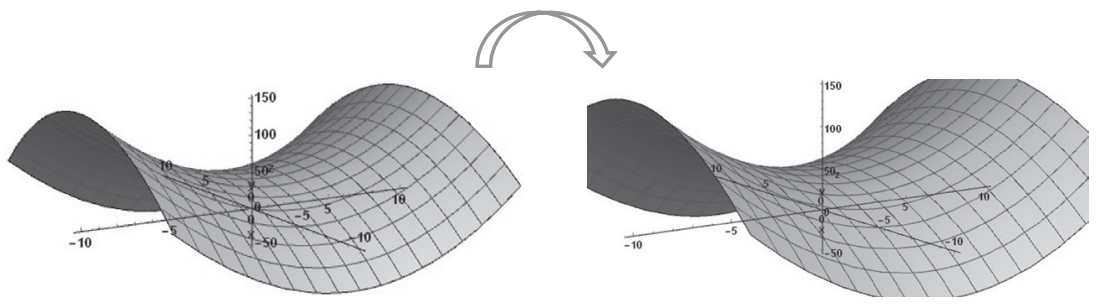


La modificación posicional ocurre cuando se mantiene el mismo tamaño y forma, pero se varía la posición. Esto tiene lugar cuando, por medio de movimientos físicos del mouse, se puede rotar el gráfico alrededor el eje z, rotarlo alrededor el plano xy y trasladarlo. Por ejemplo, la figura 6 muestra la rotación del gráfico $C A S$ alrededor del eje $z$.

Figura 6. Modificación posicional

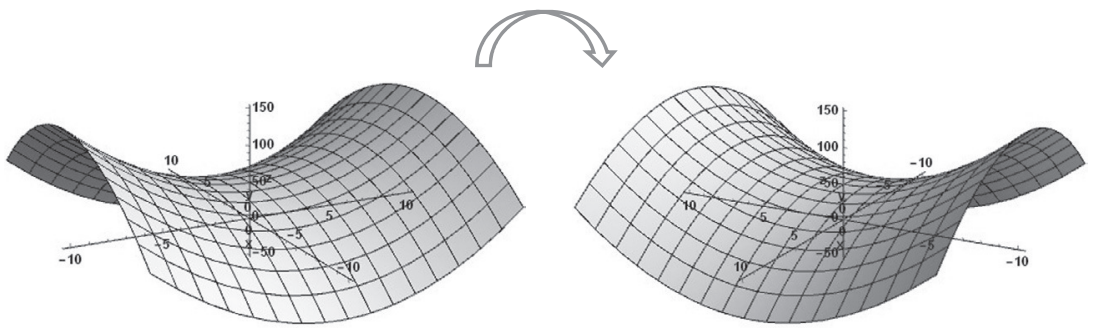

Por su parte, la modificación mereológica trata de los cortes, es decir, las intersecciones entre superficies, lo que ocurre cuando, por medio de dos comandos del software Mathematica, se generan cortes en los planos horizontales $z=k$, con los planos $x=h, y=p$ y con los planos inclinados. Por ejemplo, en la figura 7, se muestra el corte del gráfico en el plano horizontal $z=60$, obtenido cuando se escribe el comando ContourPlot $3 D[\{z==20\},\{x,-5,5\},\{y,-$ $5,5\},\{z, 0,100\}$, AxesLabel $\rightarrow\{« X », « Y », « Z »\}$, AxesOrigin $\rightarrow\{0,0,0\}$, Boxed $\rightarrow$ False $]$ y luego el comando Show para mostrar los cortes.

\section{Figura 7. Modificación mereológica}

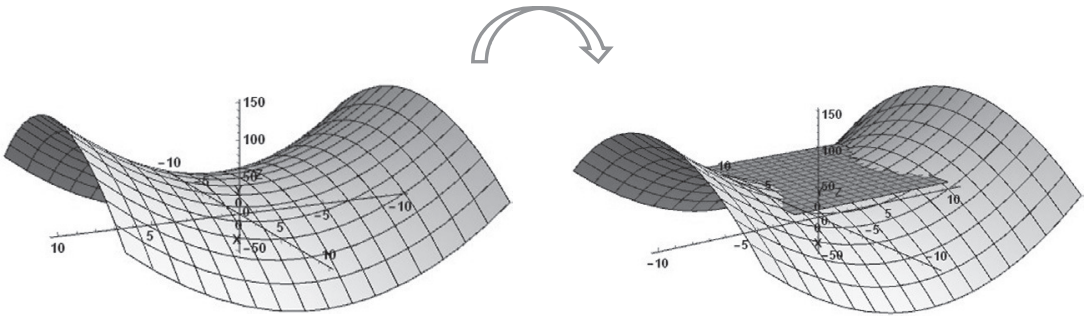

Así se levanta la hipótesis sobre la necesidad de estas aprehensiones para comprender las posibles variaciones en el registro gráfico de funciones de dos variables, puesto que hay interés en estudiar las actividades cognitivas que el estudiante moviliza para desarrollar la visualización en dicho registro, ya que las aprehensiones en este registro permiten la organización de relaciones entre unidades de representación, es decir, entre las variables visuales. 
Además, el registro gráfico permite tratamientos puramente visuales que pueden olvidarse cuando se pasa a otro registro (lengua natural, algebraica), pero que permitieron reconocer y escoger los procesos a efectuar en este otro registro para obtener la solución matemática del problema.

\section{Metodología}

La investigación se desenvolvió a través de un enfoque cualitativo y como metodología, supuestos de la Ingeniería Didáctica. Este tipo de ingeniería emergió en la didáctica de la matemática, en primer lugar, en 1982 por Chevallard y Brousseau, y, en 1989, por Artigue. Según Artigue (1995), la Ingeniería Didáctica, vista como metodología de investigación, se caracteriza por ser un esquema experimental basado en realizaciones didácticas en salón de clase; es decir, en la concepción, realización, observación y análisis de secuencias de enseńanza, concretamente, situaciones didácticas definidas en el contexto de la Teoría de las Situaciones Didácticas.

Brousseau (como se citó en Almouloud, 2007) define así la situación didáctica:

Es el conjunto de relaciones establecidas explícitamente y/o implícitamente entre un estudiante o grupo de estudiantes, un cierto medio (que contiene eventualmente instrumentos u objetos) y un sistema educativo (el profesor) para que esos estudiantes adquieran un saber construido o en construcción (p. 33).

Para la autora, esta metodología se caracteriza también, con relación a otros tipos de investigaciones basados en las experimentaciones en clase, por el registro en el cual se sitúa y por los modos que le están asociados. La Ingeniería Didáctica se sitúa en el registro de los estudios de casos, cuya validación es esencialmente interna y, fundamentalmente, en la confrontación entre análisis a priori y el análisis a posteriori.

Así, esta metodología es singular, no por los objetivos de las investigaciones llevadas a cabo, sino por las características de su funcionamiento metodológico. El análisis a priori comprende una parte descriptiva y una predictiva. $\mathrm{El}$ análisis a posteriori es realizar conclusiones en función de las relaciones que se presentan entre los objetivos establecidos a priori con las observaciones realizadas en la experimentación.

Los estudiantes trabajaron tanto con el CAS Mathematica como con sus conocimientos previos de funciones de dos variables reales. Utilizaron los comandos que permiten representar gráficamente puntos, planos y superficies 
en $R^{3}$. El tiempo de duración de las situaciones didácticas fue de tres horas cada una. Las situaciones didácticas propuestas tuvieron en común la exigencia de una discriminación de variables visuales de las formas perceptivas elementales, las cuales se imponen a primera vista para conseguir una capacidad fundamental que es la condición necesaria para toda utilización heurística de los registros gráficos: Realizar tratamientos en el registro gráfico con el fin de descubrir sobre el gráfico un procedimiento de solución.

En este artículo, se presenta la segunda situación didáctica correspondiente al segundo encuentro con los estudiantes y se analizará el trabajo desarrollado por tres grupos. Los estudiantes trabajaron en parejas y cada una fue llamada grupo. Asimismo, utilizaron una laptop y una ficha de trabajo durante el encuentro. El esclarecimiento del texto, solo cuando fuera solicitado, y la mediación del profesor-investigador se hicieron por medio de preguntas que estimularon a movilizar los conocimientos previos de los estudiantes.

\section{ResUltados y Discusión}

La continua necesidad de atender la demanda de productos variados y saludables a todo tipo de consumidores motivó a una empresa a elaborar galletas naturales y a lanzar al mercado dos tipos de ellas: la galleta integral y la galleta de avena, cuya presentación es hecha en bolsas de 24 unidades. Los costos totales de producción son de dos y tres soles por bolsa, respectivamente. La demanda (en miles de bolsas) de galletas integrales, que pueden ser vendidas cada semana, es cuatro veces la diferencia del precio del segundo producto en relación con el primero, y la demanda (en miles de bolsas) de galletas de avena es cuatro veces la diferencia del precio del primer producto con relación al doble del segundo; pero la preferencia de los consumidores por esta galleta aumenta su demanda siempre en 36 miles de bolsas.

¿Cuál será la mayor utilidad que obtiene la empresa y cuáles serían los precios de venta de cada tipo de galleta? Justifique su respuesta.

Las variables didácticas consideradas fueron tres:

- La función costo total de producción de bolsas de galletas.

- La función demanda de bolsas de galletas.

- La función utilidad, cuya representación es una función de dos variables del tipo: $f(x, y)=a x^{2}+b x y+c y^{2}+d x+e y+f$ 


\subsection{Análisis a priori}

Esa situación tiene por finalidad llevar al estudiante a comprender las nociones de valor máximo local de una función de dos variables representada por $f(x, y)=a x^{2}+b x y+c y^{2}+d x+e y+f$, permitiendo que los estudiantes movilicen sus conocimientos de plano tangente a una superficie y de derivadas parciales en un punto, dado que esos conocimientos ya fueron construidos por los estudiantes, es decir, que movilicen sus conocimientos disponibles (Robert, 1998).

Se espera que todos los grupos, después de leer el enunciado del problema, realicen la conversión del registro en lengua natural para el registro algebraico, pudiendo representar el costo total de producción con la letra $\boldsymbol{C}$, por ejemplo, el objeto función demanda, por $q=f\left(p_{1}, p_{2}\right)$, dado que, según los datos de la situación, este relaciona la cantidad de bolsas de galleta (en miles de bolsas) con los precios unitarios de cada bolsa de galleta, y el ingreso total por la venta de bolsas de galletas lo representarían por $R_{T}$. Para representar algébricamente cada uno de los objetos, los grupos podrían representarlos tanto para las galletas integrales como para las de avena, o sea, para las galletas integrales, la función de demanda de galletas podría ser representada por $q_{1}=4\left(p_{2}-p_{1}\right)$, y la función ingreso total sería representada como $R_{1}\left(p_{1}, p_{2}\right)=4\left(p_{2}-p_{1}\right) p_{1}$.

Igualmente, para las galletas de avena, la función de demanda de galletas sería representada por $q_{2}=36+4\left(p_{1}-2 p_{2}\right)$ y la función ingreso como $R_{2}\left(p_{1}, p_{2}\right)=\left[36+4\left(p_{1}-2 p_{2}\right] p_{2}\right.$. Podrían los grupos, por medio de operaciones posibles en ecuaciones y expresiones algebraicas, representar la función lucro por $l\left(p_{1}, p_{2}\right)=8 p_{1} p_{2}+52 p_{2}-4 p_{1}^{2}-4 p_{1}-8 p_{2}^{2}-108$.

Se espera que los estudiantes representen la función lucro en su forma canónica, pero observarían que no es posible y que esa representación algebraica no es conocida. Luego, suponiendo que los grupos usen el software Mathematica como medio para representar, en el registro gráfico, la función lucro (ver figura 8) pudiendo escribir, por ejemplo, el comando Plot $3 D\left[8 p_{1} p_{2}-4 p_{1}{ }^{2}\right.$ $-8 p_{2}^{2}+52 p_{2}-4 p_{1}-108,\left\{p_{1}, 0,10\right\},\left\{p_{2}, 0,10\right\}$, PlotRange $\rightarrow\{0,50\}$, AxesLabel $\rightarrow\left\{" p_{1}\right.$ "," $\left.p_{2} ", " l "\right\}$.

Suponiendo que a través de la aprehensión perceptiva, los grupos identifiquen que el registro gráfico de la función lucro es un paraboloide elíptico rotado, por la aprehensión operatoria realicen modificaciones ópticas y posicionales en dicho registro gráfico, identificando la relación de los puntos de la superficie con respecto al eje $l($ eje z) y a la curvatura de la superficie, lo que permitirá tener una percepción del posible valor máximo de la función lucro. Es más, se espera que los grupos, por medio de modificaciones mereológicas 
dentro del registro gráfico $C A S \_M A T H$, tracen planos perpendiculares al eje $l$, por ejemplo, los planos representados por $l=30 \mathrm{y} l=35$, conforme se muestra en la figura 9.

Figura 8. Registro CAS_MATH de la función lucro

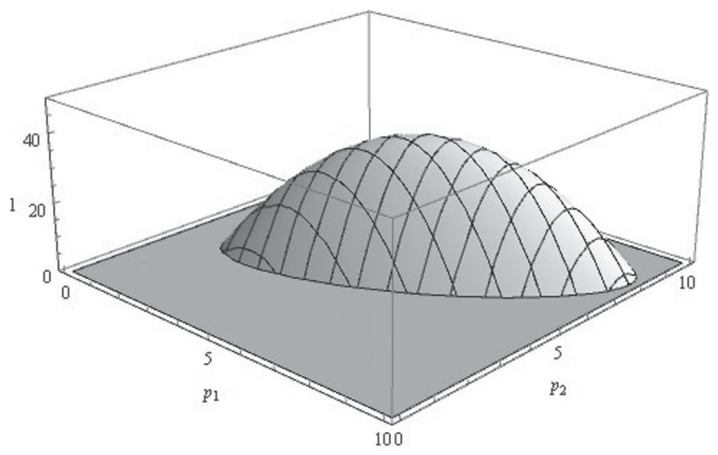

Figura 9. Modificación mereológica de un registro gráfico

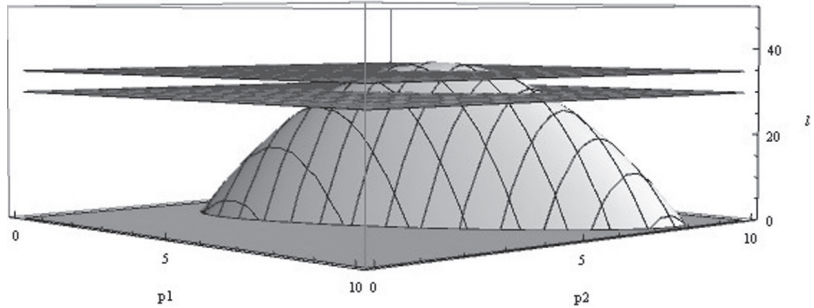

Los estudiantes podrían conjeturar y formular que, en el valor máximo, la superficie está debajo completamente del plano perpendicular al eje $l$, y que el valor máximo de la superficie se localiza en el punto donde el plano perpendicular al eje $l$ es tangente a la superficie.

\subsection{Análisis a posteriori}

El grupo 1 representó la función lucro en el gráfico $C A S$ para tener una aprehensión perceptiva del valor máximo, por medio de modificaciones ópticas, es decir, el grupo presionó la tecla Ctrl y clicó el botón izquierdo del mouse, movió físicamente el mouse y manipuló el gráfico, de tal manera que ampliando y reduciendo el registro gráfico percibió la posición de la superficie en relación con los ejes coordenados orientados positivamente, como muestra la figura 10, tal como se había supuesto en el análisis a priori. 
Figura 10. Modificación óptica, posicional y mereológica en el registro

$C A S$ - grupo 1

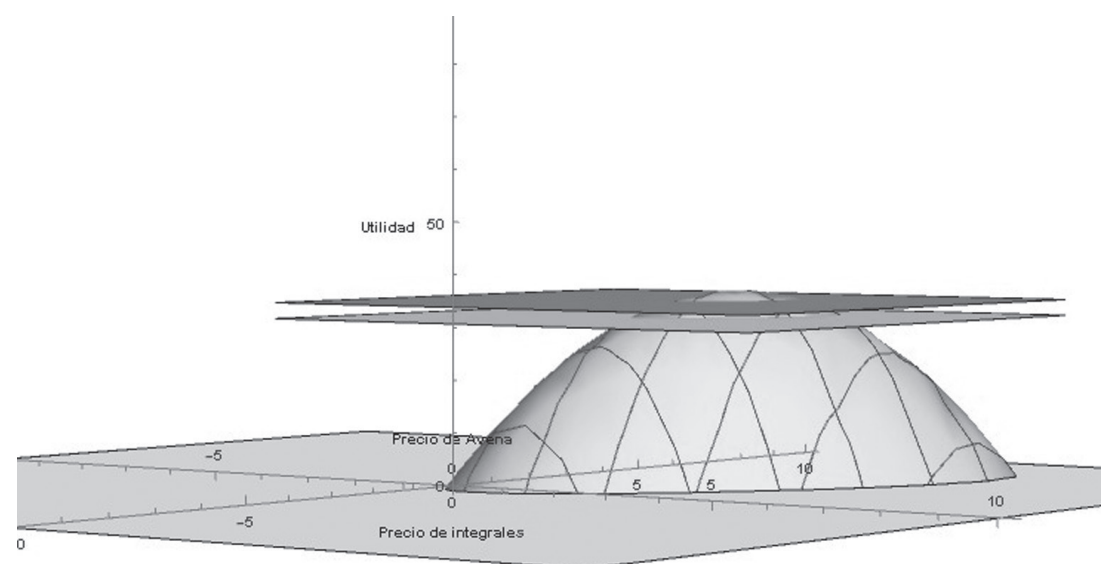

Esto significa que el grupo desarrolló su aprehensión operatoria, la cual produce la fecundidad intuitiva del registro gráfico $C A S$, pero esta (aprehensión) es dirigida por la aprehensión perceptiva, es decir, empezó a desarrollar su proceso de visualización para identificar el valor máximo de la función lucro. Más aún, se afirma que existe conexión entre la aprehensión operatoria y discursiva, dado que el estudiante hizo una interpretación discursiva de los elementos visuales del registro gráfico $C A S$, conforme se muestra en la figura 11. Esto significa que el grupo desarrolló su proceso heurístico para resolver la situación didáctica.

Figura $11^{1}$. Interpretación discursiva de elementos visuales.

$$
\begin{aligned}
& \text { Entonces pormando la ccuación cirl plenso tangente } \\
& \text { obtendremos: } P=(x, y, z) \text { y } P_{0}=\left(x_{0}, y_{0}, z_{0}\right) \in P_{T} \\
& P_{i}: \vec{n}_{p r \cdot}\left[P-P_{0}\right]=0 \text {, } \\
& \text { Pew la nortwal al ins tangente es igual a. } \\
& \bar{n}_{P T}=\left(\frac{\partial U_{\left(x_{0} ; x_{0}\right.}}{\partial x} \frac{\partial U_{\left(x_{0}, x_{0}\right)}}{\partial y}, 1\right)=(0,0,1)
\end{aligned}
$$

\footnotetext{
1 Entonces formando la ecuación del plano tangente obtendremos $P=(x, y, z)$ y $P_{0}=\left(x_{0}, y_{0}, z_{0}\right) \in P_{T}$. $P_{T}: \vec{n}_{P_{T}}\left[P-P_{0}\right]=0$. Pero la normal al plano tangente es igual a $\vec{n}_{P_{T}}=\left(\frac{\partial U}{\partial x}\left(x_{0}, y_{0}\right), \frac{\partial U}{\partial y}\left(x_{0}, y_{0}\right), 1\right)=(0,0,1)$.
} 
La manera de ver de este grupo corresponde a la aprehensión global cualitativa, lo que ayudó a su aprendizaje sobre el uso de la derivada parcial para encontrar el valor máximo y el valor de máximo de una función de dos variables reales, cuya representación algebraica no era conocida.

El grupo 2, en un primer momento, basándose en su aprehensión perceptiva, ve el registro gráfico $C A S$ de manera icónica, pues relaciona los puntos de la superficie con el eje $l$, variable que representa la función lucro. En un segundo momento, realiza modificaciones ópticas y posicionales en el registro gráfico $C A S$ para representar puntos en la superficies representada por $l$. Esto nos permite afirmar que el grupo comienza a identificar una asociación entre dos valores numéricos, es decir, lo observado por el grupo son asociaciones (puntos - ternas ordenadas). Este es un hecho que no habíamos supuesto en el análisis a priori, lo que significa que la mirada del grupo es también local y relaciona un punto con una terna ordenada, conforme la figura 12 .

Figura 12. Representación gráfica de un punto - grupo 2

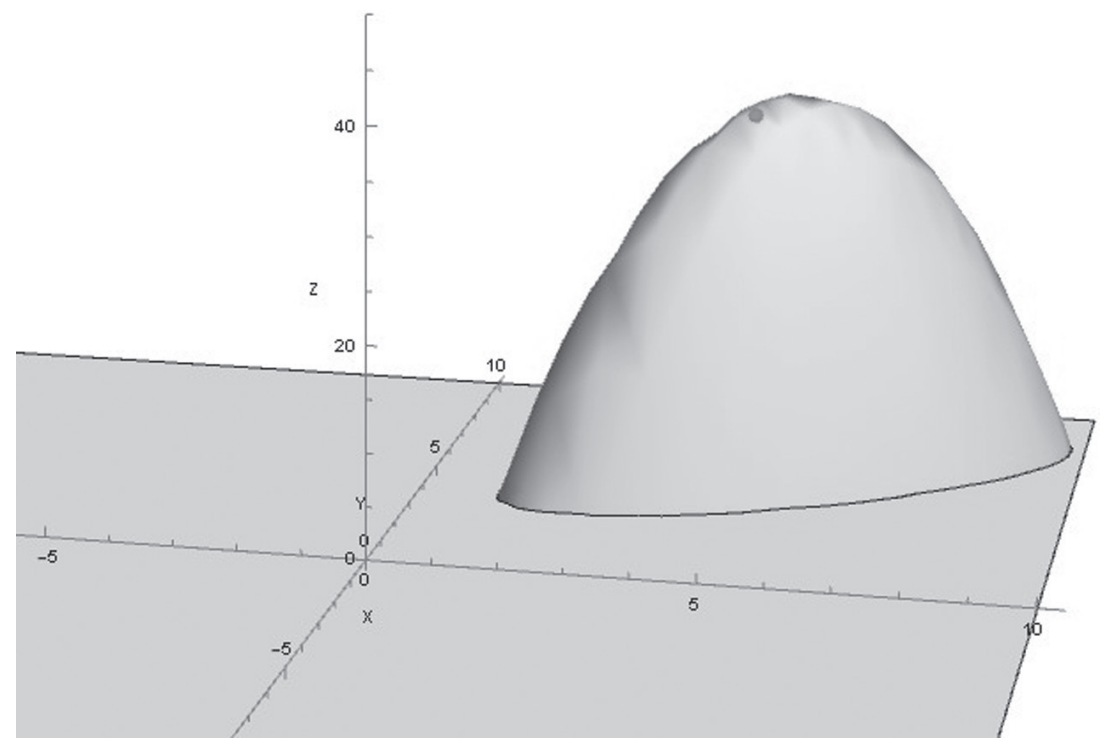

Ya, en un segundo momento, el grupo 2, por medio de modificaciones ópticas y mereológicas, representa gráficamente los planos representados por $z=35, z=40$, percibiendo la posición de la superficie en relación con el eje $z$, conforme se muestra en la figura 13. 
Figura 13. Modificación óptica y mereológica en el registro CAS - grupo 2.

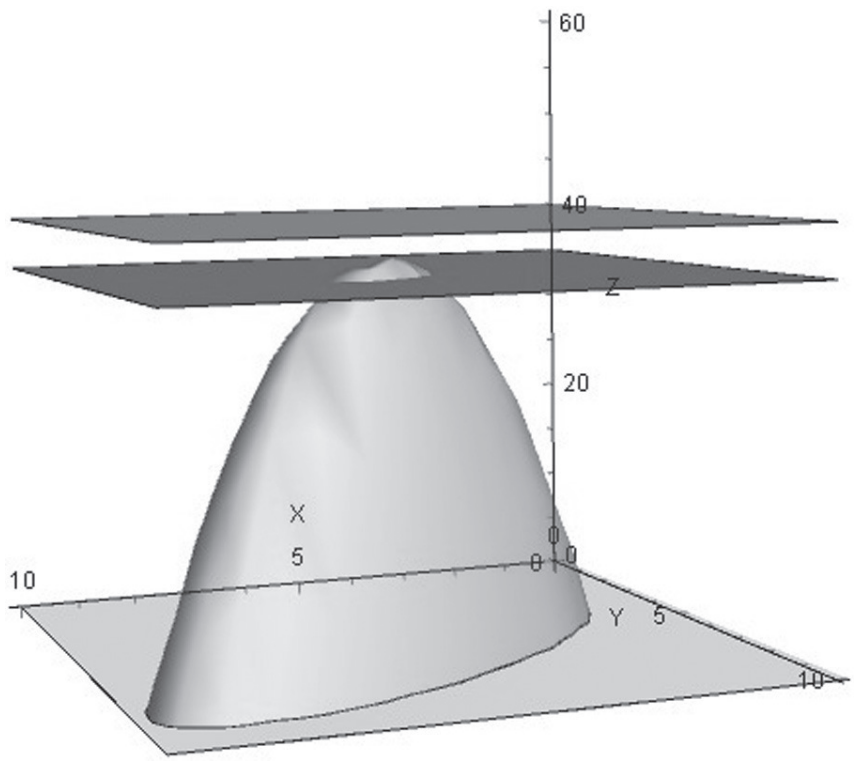

Es por la aprehensión operatoria y como muestra la figura 14 que el grupo siente la necesidad de usar las derivadas parciales para encontrar el valor máximo local de la función lucro.

Figura 14. Interpretación discursiva del plano tangente a la superficie - grupo 3

\footnotetext{
Como el plano tangente va hacer papacelo he

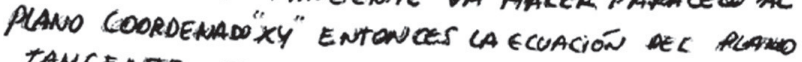
TANGENTE TENDRIA CA FORMA $z=z_{0} y z-z_{0}=0$ POR LO TANTO$$
\text { Pि } \frac{\partial \mu^{u}}{\partial x}\left(x_{0}, y_{0}\right) \cdot\left(x-x_{0}\right)+\frac{\partial y}{\partial y}\left\{x_{0}, y_{0}\right) \cdot\left(y-y_{0}\right)=z-z_{0}
$$

Y POR lO TANTO lOS coEFICIENTES, QUE SON LAS DERIVADAS PARCALESTENIRIAN QUE TOMAR EL VALOR DE CERO, PARA QUE LA EUACIÓ́ DEC PLANO TAN GENTE SEA $Z=Z_{0}$
} 
Esto significa que el grupo consigue pasar de una aprehensión local a una aprehensión global cualitativa. Esta última la habíamos supuesto en el análisis a priori.

El grupo 3, a diferencia del grupo 1 y del grupo 2, representó gráficamente la función lucro en el registro gráfico CAS_MATH, conforme se había supuesto en el análisis a priori. En un primer momento, este grupo vio el gráfico de manera icónica, dado que, el registro gráfico se presta para que el grupo identifique una analogía con cambio de posición, es decir, estar más alto, estar más bajo. Se afirma que debido al parecido del registro gráfico con el perfil de una colina, el grupo en relación con un nivel horizontal observa desplazamientos de subida para identificar el valor máximo de la función lucro. En un segundo momento, el grupo identifica una asociación entre dos valores numéricos (puntos-ternas ordenadas), lo que nos indica que esta asociación se limita a la aplicación de una simple regla de codificación, pues cada punto de intersección de un octante puede ser asociado a una terna de números, conforme muestra la figura 15. Se afirma que, sobre este grupo, si bien realiza modificaciones en el registro gráfico $C A S \_M A T H$, su manera de ver se limita a operaciones locales y sucesivas de codificación, que es equivalente a una lectura del gráfico. La aprehensión perceptiva subordina al tipo de tratamiento que puede ser desarrollado en el registro gráfico.

Figura 15. Representación de ternas ordenadas en el gráfico CAS_MATH - grupo 3

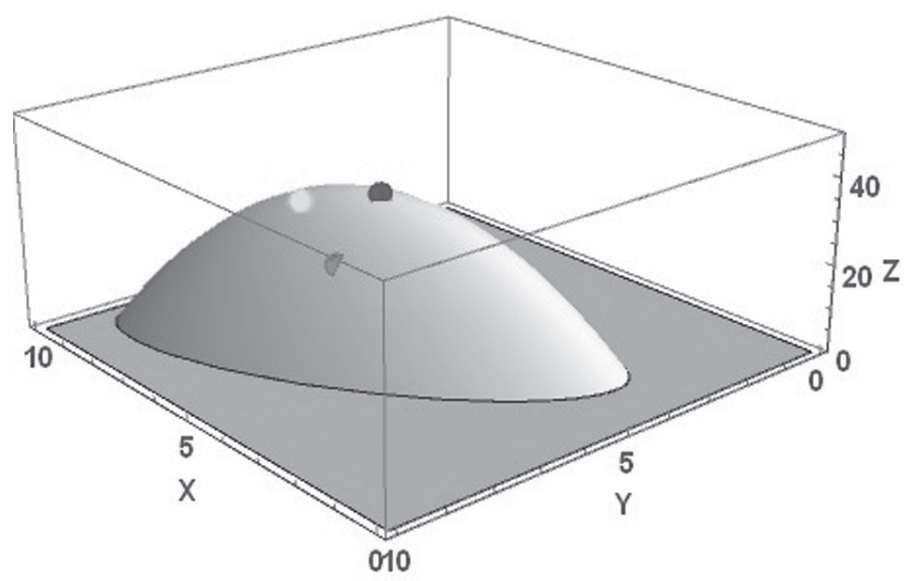


Dicho lo anterior, se afirma que el grupo fue guiado por la aprensión perceptiva del registro gráfico CAS, a ver este registro de modo local e icónico. Este grupo no pasó a una aprehensión global cualitativa, hecho que no se había supuesto en el análisis a priori.

\section{Conclusiones}

Las aprehensiones en el cálculo de funciones de dos variables presentan otro modo de ver la enseńanza del cálculo. El uso extensivo de los registros gráficos y su uso en la solución de problemas fue muy exigente y completamente nuevo para los estudiantes

Se considera que la aprehensión operatoria en el registro gráfico CAS, tales como las modificaciones ópticas, posicional y mereológica, son operaciones fundamentales en el registro gráfico, porque brindan la posibilidad de hacer modificaciones en dicho registro y relacionarlas con propiedades matemáticas por medio de la aprehensión discursiva para, así, tener otra manera de ver la enseñanza del cálculo de funciones de varias variables.

Asimismo, se piensa que la articulación de las aprehensiones, en el registro gráfico $C A S$, permite a los alumnos conjeturar las propiedades de las funciones de dos variables y de las derivadas parciales cuando, por ejemplo, el estudiante aplica esas nociones a problemas de optimización.

Se recomienda el uso del software Mathematica como medio para facilitar el proceso de visualización, puesto que permite que el usuario, por medio de la articulación de la aprehensión, perceptiva, operatoria y discursiva, movilice y realice conexiones con los conocimientos de los elementos del cálculo de dos variables que emergen en la representación gráfica de las funciones de dos variables.

El desarrollo de las tres maneras de ver apunta hacia una dirección en la enseñanza y aprendizaje del cálculo de varias variables en clase, las cuales van de la puntual a la aprehensión global cualitativa, que es específica de las matemáticas y exige un cambio completo del funcionamiento cognitivo, por parte del estudiante de ingeniería.

La situación didáctica propuesta favoreció la participación efectiva del estudiante en el proceso de apropiación de los conocimientos y saberes de la derivada parcial. Los errores identificados, gracias a la situación propuesta, son puntos de apoyo de aprendizaje, pues su superación es fuente de aprendizaje. Además, nos permitió darnos cuenta de las concepciones de los estudiantes y la construcción del significado de la derivada parcial. 


\section{REFERENCIAS BIBLIOGRÁFICAS}

Almouloud, S. (2007). Fundamentos da Didática da Matemática. Curitiba: Universida de Federal do Paraná.

Artigue, M. (1995). Ingeniería Didáctica. En P. Gómez (ed.), Ingeniería Didáctica en Educación Matemática. Un esquema para la investigación y la innovación en la enseñanza y el aprendizaje de las matemáticas (pp. 33-59). Bogotá, Colombia: Grupo Editorial Iberoamérica. Recuperado de https://core. ac.uk/download/pdf/12341268.pdf.

Duval, R. (1994). Les différents fonctionnements d'une figure dans une démarche géométrique. Repères-IREM, 17, 121-138. Recuperado de http://www. univ-irem.fr/exemple/reperes/articles/17_article_119.pdf.

Duval, R. (1995). Sémiosis et pensée humaine: registres sémiotiques et apprentissages intellectuels. Berna: Editorial Peter Lang.

Duval, R. (1996). Quel cognitif retenir en didactique des mathématiques? Recherches en Didactique des Mathématiques, 16(3), 349-382.

Duval, R. (1999). Representation, vision and visualization: Cognitive functions in mathematical thinking. Basic issues for learning. Proceedings of the Annual Meeting of the North American Chapter of the International Group for the Psychology of Mathematics Education (pp 3-26). Cuernavaca, México. Recuperado de https://files.eric.ed.gov/fulltext/ED466379.pdf

Duval, R. (2003). Décrire, visualiser ou raisonner : quels apprentissages premiers de l'activité mathématique? Annales de Didactique et de Sciencies Cognitives, (8), 13-62.

Duval, R. (2004). Los problemas Fundamentales en el Aprendizaje de la Matemáticas $y$ las Formas Superiores en el Desarrollo Cognitivo. Traducción de Myriam Vega Restrepo. Colombia: Universidad del Valle, Instituto de Educación y Pedagogía, Grupo de Educación Matemática.

Guzmán, M. (1996). El rincón de la pizarra. Ensayos de visualización en análisis matemático. España: Pirámide, S.A.

Ingar, K. (2014). A visualização na Aprendizagem dos Valores Máximos e Minimos Locais da Função de DuasVariáveisReais. (Tesis doctoral en Educación Matemática). Pontifícia Universidade Católica de São Paulo, São Paulo, Brasil.

Ingar, K. y Silva, M. (2015) A visualização de valores máximos e mínimos de funçōes de duasvariáveis. En N. Amado y S. Carreira (eds.), Proceedings of the 12th International Conference on Technology in Mathematics Teaching. University of Algarve, Faro, Portugal. Recuperado de https://sapientia. ualg.pt/handle/10400.1/6081. 
Kabael, T. (2009).The effects of the function machine on students 'understanding levels and their image and definition for the concept of function. En Swars, S. L., Stinson, D.W. y Lemons-Smith, S. (eds.), Proceedings of the 31 sr annual meeting of the North American Chapter of the International Group for the Psychology of Mathematics Education. Atlanta, GA: Georgia, State University, USA.

Kashefi, H., Zaleha, I. y Mohammad, Y. (2010). Obstacles in the Learning of Twovariable Functions through Mathematical Thinking Approach. Procedia Social and Behavioral Sciences, 8, 173-180. https://doi.org/10.1016/j. sbspro.2010.12.024.

Montiel, M., Wilhelmi, M., Vidakovic, D. y Elstak, I. (2009). Using the ontosemiotic approach to identify and analyze mathematical meaning when transiting between different coordinate systems in a multivariate context. Educational Studies in Mathematics, 72(2), 139-160. https://doi. org/10.1007/s10649-009-9184-2

Robert, A (1998). Outils d'analyses des contenus mathématiques à enseigner au lycée et à l'université. Recherches en didactique des mathématiques, 18(2), 139-190.

Thomas, G.B. (2005). Cálculo. Varias variables. México: Pearson Educación. Recuperado de https://www.u-cursos.cl/usuario/7c1c0bd54f14c0722cefc 0fa25ea186d/mi_blog/r/Thomas_Calculo_Varias_Variables_\%28Thomas \%29_-_11o_Edicion.pdf

Trigueros, M. y Martínez-Planell, R. (2010). Geometrical representations in the learning of two-variable functions. Educational Studies in Mathematics, 73(1), 3-19. https://doi.org/10.1007/s10649-009-9201-5

Trigueros, M. y Martínez-Planell, R. (2011). How are graphs of two variable functions taught? En L. Wiest y T. Lamberg (eds.), Proceedings of the 33rd Annual Conference of the North America Chapter of the International Group for the Psychology of Mathematics Education. Reno, Nevada, USA. Recuperado de https://www.pmena.org/pmenaproceedings/PMENA\%20 33\%202011\%20Proceedings.pdf.

Yerushalmy, M. (1997).Designing representations: Reasonning about functions of two variables. Journal for Research in Mathematics Education, 28(4), 431-466. https://doi.org/10.2307/749682

Zimmermann, W. y Cunningham, S. (eds.) (1991). Visualization in Teaching and Learning Mathematics, MAA Notes 19. 